\title{
Development of dynamic aurora pulsed laser deposition equipped with reflection high-energy electron diffraction and effects of magnetic fields on room-temperature epitaxial growth of $\mathrm{NiO}$ thin film
}

\author{
Takahiko KAWAGUCHI ${ }^{1}$, Mayu YOSHIDA ${ }^{1}$, Naonori SAKAMOTo ${ }^{1,2}$, Kazuo SHINOZAKI ${ }^{2,3}$, \\ Hisao SUZUKI ${ }^{1,2,4}$ and Naoki WAKIYA ${ }^{1,2,4, \dagger}$ \\ ${ }^{1}$ Graduate School of Integrated Science and Technology, Department of Engineering, Shizuoka University, \\ 3-5-1 Johoku, Naka-ku, Hamamatsu 432-8561, Japan \\ ${ }^{2}$ Research Institute of Electronics, Shizuoka University, 3-5-1 Johoku, Naka-ku, Hamamatsu 432-8561, Japan \\ ${ }^{3}$ School of Materials and Chemical Technology, Tokyo Institute of Technology, \\ 2-12-1 0-okayama, Meguro-ku, Tokyo 152-8550, Japan \\ ${ }^{4}$ Graduate School of Science and Technology, Shizuoka University, 3-5-1 Johoku, Naka-ku, Hamamatsu 432-8561, Japan
}

\begin{abstract}
Pulsed laser deposition (PLD) with an electromagnet (dynamic aurora PLD) and reflection high-energy electron diffraction (RHEED) was developed by installing an 11-tuned solenoid coil in a vacuum chamber. This method achieved thin film deposition under a magnetic field of up to $120 \mathrm{mT}$ using de current of $1,200 \mathrm{~A}$. The electromagnet is a solenoid coil, which produces a magnetic field that is proportional to an electric field without saturation of the magnetic field. This PLD method enables in situ observation of the crystal structure of the thin film surface after turning the electromagnet off, but without breaking vacuum. Many reports have described studies of PLD in a magnetic field. For most such studies, permanent magnets have been used to apply a magnetic field to the plume. Nevertheless, when doing so, carrying out in-situ observation using RHEED is difficult because the magnetic field deflects the electron beam. This case study examined effects of magnetic field application to the plume on the in-plane orientation and resistivity of a $\mathrm{NiO}$ thin film deposited on a $\mathrm{MgO}(001)$ single crystal at room temperature $\left(25^{\circ} \mathrm{C}\right)$. Results demonstrate that the in-plane orientation (epitaxial growth) is unchanged, whereas thin film resistivity decreases exponentially with the magnetic field intensity during deposition. These results suggest that the $\mathrm{Ni}^{3+}$ ion concentration can be expected to increase exponentially with the magnetic field during deposition. The room-temperature resistivity of the NiO thin film deposited under a $120 \mathrm{mT}$ magnetic field was $112 \Omega \mathrm{cm}$.

(C2021 The Ceramic Society of Japan. All rights reserved.
\end{abstract}

Key-words : Dynamic aurora PLD, Electromagnet, NiO thin film, Resistivity, RHEED

[Received November 25, 2020; Accepted January 22, 2021]

\section{Introduction}

For pulsed laser deposition (PLD) use in preparing thin films, a target surface is irradiated by a focused pulsed laser beam to generate a plasma "plume" that includes electrons, cations, neutral atoms, and their clusters. The respective lifetimes of electrons and cations are very short because of recombination, but their lifetimes can be extended drastically by applying a magnetic field to the plume. ${ }^{1)}$ In doing so, cations with high energy are deposited on the substrate surface. The first report of PLD in a

Corresponding author: N. Wakiya; E-mail: wakiya.naoki@ shizuoka.ac.jp

\$ Preface for this article: Dol http://doi.org/10.2109/jcersj2. 129.P7-1 magnetic field was presented by Kobayashi et al., ${ }^{2)}$ who put a rare-earth permanent magnet on the substrate surface. Since that report, numerous researchers have examined the effects of magnetic field application to a plume. ${ }^{3)}$ Typically reported effects on PLD of a magnetic field applied using permanent magnets or electromagnets have included (i) elimination of droplets, ${ }^{4), 5)}$ (ii) lowering of the crystallization temperature, improvement of crystallinity, and enhancement of thin film properties, ${ }^{6)-8)}$ (iii) change of thin film morphology, ${ }^{9-11)}$ and (iv) magnetic-field-induced phase separation. ${ }^{12)-14)}$

Reflection high-energy electron diffraction (RHEED) has been used extensively for in-situ observation of thin film surfaces. In fact, PLD is very commonly used along with RHEED. Nevertheless, using RHEED under a magnetic field is difficult because of electron beam deflection. 
Therefore, if a magnetic field is applied using a permanent magnet, then the following procedure is necessary: (1) breaking vacuum, (2) removing the permanent magnet, (3) evacuating the vacuum chamber again, and (4) conducting RHEED observation. However, exposure to air brings about surface contamination that is expected to cause surface structure alteration. Therefore, RHEED observation for aurora PLD requires magnetic field application using an electromagnet. In this work, the term "in situ observation" is used to mean "observation without breaking vacuum," not "real-time observation during deposition." The use of an electromagnet offers compatibility between magnetic field application during deposition and in-situ RHEED observation if the magnetic field is turned off during observation. Furthermore, the electromagnet can be used to produce a "variable magnetic field" during deposition by controlling the electromagnet current. No report of the relevant literature describes PLD development simultaneously with an electromagnet and RHEED. Salient benefits of RHEED observation are that it supports crystal structure analysis for ultrathin films and growth mode analysis for the initial stage of film growth, which are difficult to accomplish using X-ray diffractometer (XRD) alone. The use of an electromagnet instead of a permanent magnet is expected to realize a new crystal structure and functionality for oxide and semiconductor thin films. ${ }^{12)}$

Generally speaking, substrate heating is necessary to achieve epitaxial growth of oxide ceramics. However, epitaxial growth of $\mathrm{NiO}$ thin films has been reported even at room temperature. ${ }^{6), 15), 16)}$ Although the room-temperature resistivity of pure stoichiometric $\mathrm{NiO}$ crystals is as high as $10^{13} \Omega \mathrm{cm}$, the resistivity of $\mathrm{NiO}$ is reportedly low from the perspective of defect chemistry. ${ }^{17)}$ Usual $\mathrm{NiO}$ is not stoichiometric. It includes an excess of oxygen that is accommodated by nickel vacancies. To preserve overall electrical neutrality in the crystal, some $\mathrm{Ni}^{2+}$ ions must be converted to $\mathrm{Ni}^{3+}$ ions, which are then responsible for the conduction of $\mathrm{NiO} .{ }^{18)}$ For $\mathrm{NiO}$ thin films deposited by sputtering, thin film resistivity reportedly changes with substrate temperature during deposition. This result has demonstrated that the $\mathrm{Ni}^{3+}$ ion concentration changes with substrate temperature because sputtering is far from an equilibrium state of the material. ${ }^{19)}$

Because thin film deposition under a magnetic field (dynamic aurora PLD) at room temperature is also regarded as far from an equilibrium state, the resistivity of roomtemperature grown $\mathrm{NiO}$ thin films is expected to be controllable by the magnetic field that is used during deposition. Therefore, this work serves two purposes: development of PLD with an electromagnet (dynamic aurora PLD) and RHEED; and assessment of the effects of magnetic field application on the crystal structure and examination of ceramic thin film properties. As an example of the second purpose, the effects of magnetic field application during deposition on a crystal structure and electrical conductivity of a room-temperature deposited $\mathrm{NiO}$ thin film were examined.

\section{Development of dynamic aurora PLD with RHEED}

Figure 1 portrays a schematic drawing of dynamic aurora PLD apparatus equipped with RHEED. The distance between the target top and the substrate bottom is $55 \mathrm{~mm}$. In this gap, a $50 \mathrm{~mm}$-thick electromagnet was installed under the RHEED beamline. Figure 2(a) presents a photograph of an electromagnet (solenoid coil) made of an 11-tuned hollow conductor and its stand. The hollow conductor cross-section is $10 \mathrm{~mm} \times 10 \mathrm{~mm}$ square copper having a $6 \mathrm{~mm} \phi$ through hole. The hollow conductor surface was covered with polyimide tape and was rolled up. Figure 2(b) presents a photograph of an electromagnet installed in the vacuum chamber. To prevent charging by the RHEED electron beam, the electromagnet surface was covered with conductive aluminum tape. For this electromagnet, dc current of up to $1,200 \mathrm{~A}$ and $6 \mathrm{~L} / \mathrm{min}$ of cooling water were applied. Figure 3 presents the relation between the magnetic field and the electromagnet current. The magnetic field was measured at the center of the electromagnet's upper edge. This figure shows a linear relation because the electromagnet is a solenoid coil without saturation of the magnetic field. As this figure shows, a $120 \mathrm{mT}$ magnetic field is obtained when using 1,200 A of dc current.

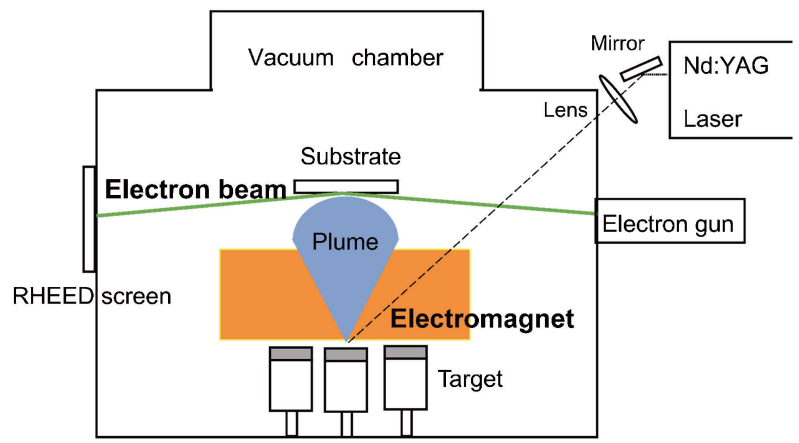

Fig. 1. Schematic drawing of dynamic aurora PLD equipped with RHEED.
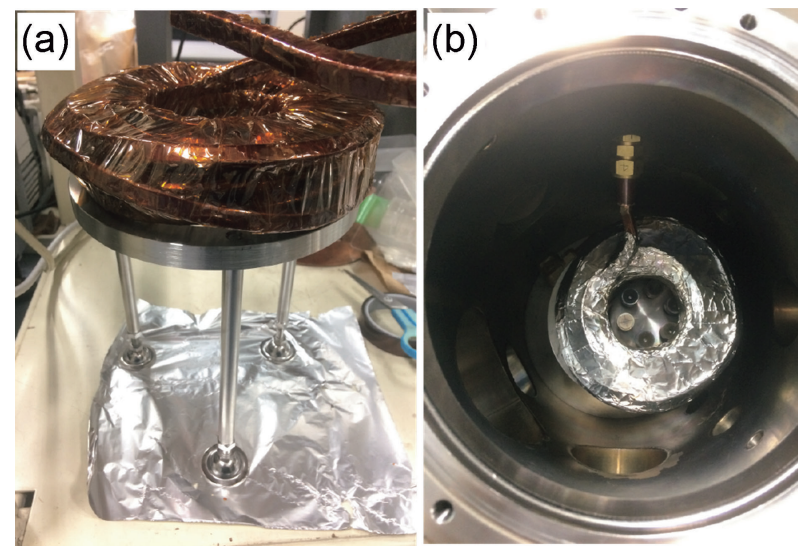

Fig. 2. Photographs of (a) an electromagnet (solenoid coil) made of an 11-tuned hollow conductor on a stand and (b) an electromagnet installed in the vacuum chamber. The electromagnet surface was covered with aluminum tape to prevent charging by the RHEED electron beam. 


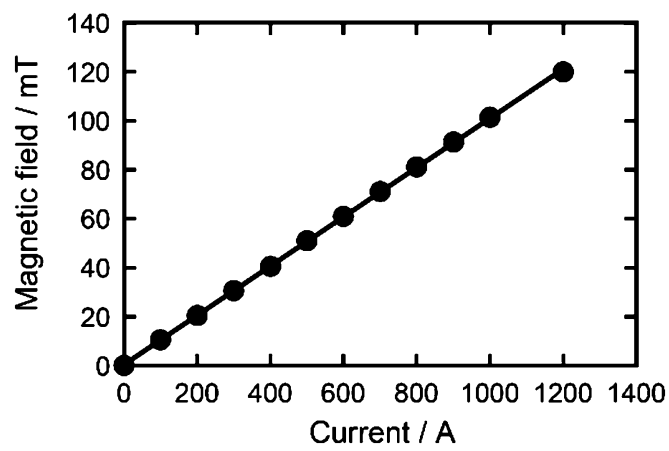

Fig. 3. Relation between the magnetic field and electromagnet current.

Table 1. Deposition conditions of $\mathrm{NiO}$ thin films

\begin{tabular}{ll}
\hline Laser & $\mathrm{Nd}: \mathrm{YAG}$ laser $(\lambda=266 \mathrm{~nm})$ \\
Repetition rate $/ \mathrm{Hz}$ & 10 \\
Target & $\mathrm{NiO}$ \\
Substrate & $\mathrm{MgO}(001)$ \\
Fluence $/ \mathrm{J} / \mathrm{cm}^{2}$ & 2.0 \\
Magnetic field $/ \mathrm{mT}$ & $0-120$ \\
Substrate temperature $/{ }^{\circ} \mathrm{C}$ & 25 \\
Oxygen pressure during deposition $/ \mathrm{Pa}$ & $1.33 \times 10^{-2}$ \\
\hline
\end{tabular}

\section{Experimental}

Using this dynamic aurora PLD with a Nd:YAG laser $(\lambda=266 \mathrm{~nm}), \mathrm{NiO}$ thin films were deposited on $\mathrm{MgO}(001)$ substrates. For this study, thin film deposition was done at room temperature under a $0-120 \mathrm{mT}$ magnetic field. A sintered $\mathrm{NiO}$ ceramic disc was used as the target. Detailed deposition conditions are presented in Table 1.

The NiO thin films were characterized based on RHEED observations conducted after turning off the electromagnet, but without breaking vacuum. The electron gun was operated at $20 \mathrm{kV}$ with an incident angle of around $3^{\circ}$. Then the NiO thin film was taken out of the vacuum chamber. Following measurement, it was carried out in air at room temperature. The film thickness was measured using an X-ray fluorescence spectrometer (Minipal; Malvern Panalytical, UK) equipped with the FP multisoftware module. The crystal structure and pole figure of the thin film were examined using a high-resolution XRD (X'pert MRD; Malvern Panalytical, UK). The NiO thin film resistivity was measured using a resistance meter (4339B; Agilent Technologies Inc., USA).

\section{Results and discussion}

Figure 4 portrays the change of the $\mathrm{NiO}$ thin film deposition rate according to changes of the applied magnetic field during deposition. The film thickness was measured at the center of the thin film. As this figure shows, the deposition rate increases almost linearly with the magnetic field intensity. Applying a magnetic field to the plume focuses the plume because the Lorentz force applied to the flying cations serves as a centripetal force. The high deposition rate is a benefit of applying the magnetic field to the plume.

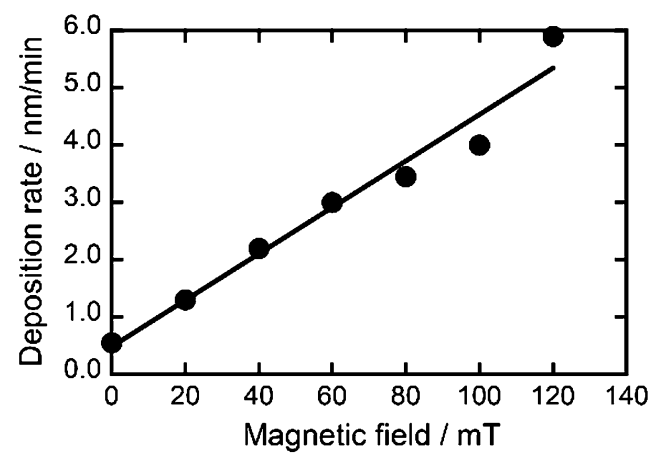

Fig. 4. Change of the $\mathrm{NiO}$ thin film deposition rate at room temperature with an applied magnetic field.
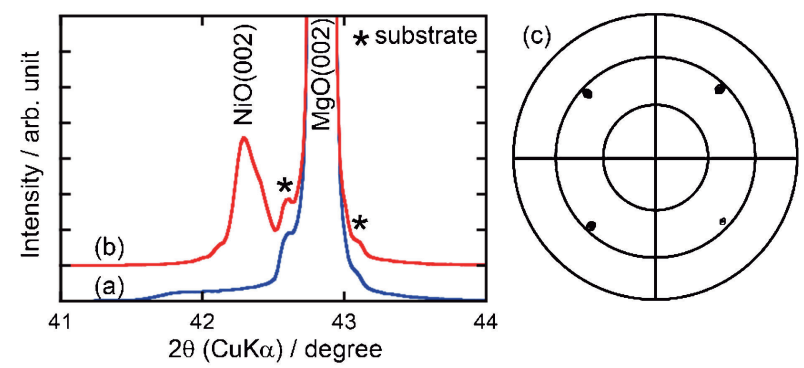

Fig. 5. XRD patterns of $\mathrm{NiO}$ thin film deposited at room temperature on $\mathrm{MgO}(001)$ under magnetic fields of (a) 0 and (b) $100 \mathrm{mT}$. (c) (111) X-ray pole figure of $\mathrm{NiO}$ thin film deposited under a magnetic field of $100 \mathrm{mT}$.

Figures 5(a) and 5(b) respectively portray XRD patterns of $\mathrm{NiO}$ thin films deposited at room temperature on $\mathrm{MgO}(001)$ in magnetic fields of 0 and $100 \mathrm{mT}$. The left panel of the figure shows two peaks marked "*" derived from the $\mathrm{MgO}(001)$ substrate. Figure 5(b) shows that a film deposited under a magnetic field of $100 \mathrm{mT}$ exhibits a 001 orientation. No such peak is observed for Fig. 5(a). However, a broad peak is observed around at $42^{\circ}$ at $2 \theta$. This broad peak is expected to indicate that the NiO film is crystalline but composed of nanocrystals. Figure 5(c) shows a (111) X-ray pole figure of $\mathrm{NiO}$ thin film deposited under a magnetic field of $100 \mathrm{mT}$. Four poles are observed at every $90^{\circ}$ in $\phi$ at $54^{\circ}$ in $\varphi$, which proves epitaxial growth of the $\mathrm{NiO}$ thin film on $\mathrm{MgO}(001)$.

Figures 6(a) and 6(d) respectively depict RHEED images of $\mathrm{MgO}(001)$ single-crystal from [100] and [110] azimuth. Figures 6(b) and 6(e) respectively show those of $\mathrm{NiO}$ thin film deposited under a magnetic field of $0 \mathrm{mT}$ from [100] and [110] azimuth. Figures 6(c) and 6(f) respectively show those of $\mathrm{NiO}$ thin film deposited under a magnetic field of $100 \mathrm{mT}$ from [100] and [110] azimuth. In Figs. 6(b) and 6(e), a streak pattern can be observed clearly. This result agrees with the consideration that the $\mathrm{NiO}$ thin film deposited without application of a magnetic field is crystalline, as described in Fig. 5(a). These RHEED images depict that the in-plane orientation of $\mathrm{NiO}$ thin film is unchanged between 0 and $100 \mathrm{mT}$, and that epitaxial growth on $\mathrm{MgO}(001)$ is realized. This in-plane orientation is observed for thin films deposited under 120 

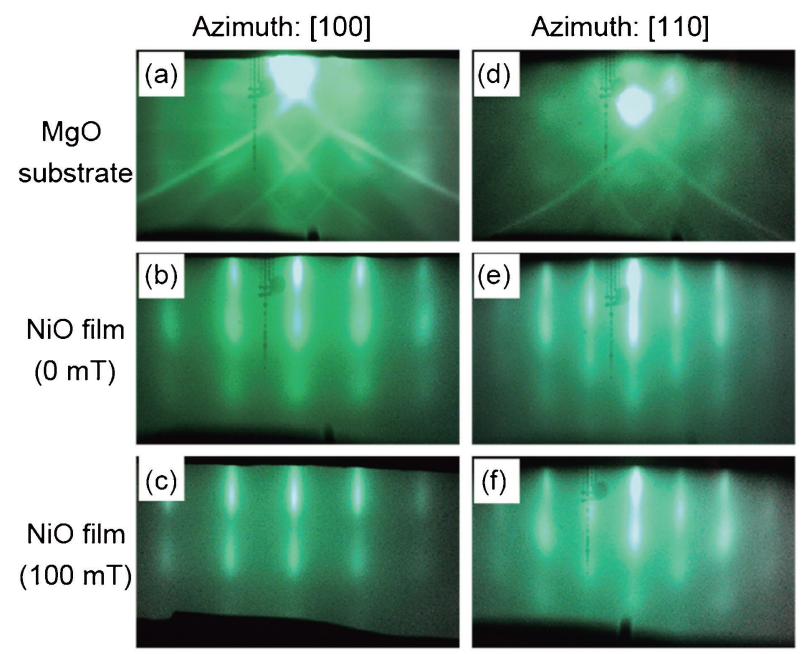

Fig. 6. (a) and (d) RHEED images of $\mathrm{MgO}(001)$ single-crystal from [100] and [110] azimuth. (b) and (e) RHEED images of $\mathrm{NiO}$ thin film deposited under a magnetic field of $0 \mathrm{mT}$ from [100] and [110] azimuth. (c) and (f) RHEED images of NiO thin film deposited under a magnetic field of $100 \mathrm{mT}$ from [100] and [110] azimuth.

$\mathrm{mT}$, although the images are not shown here. As presented in Fig. 5(a), the thin film deposited without applying magnetic field appears to be amorphous from XRD, whereas Figs. 6(b) and 6(e) show epitaxial growth. This result suggests that the crystallinity of $\mathrm{NiO}$ thin film deposited without applying a magnetic field during deposition is low, but in-plane crystallinity can be observed using RHEED. Kobayashi et al. deposited a $\mathrm{NiO}$ thin film on $\mathrm{MgO}(001)$ substrate at room temperature without applying a magnetic field. They found that the $\mathrm{NiO}$ thin film crystallinity depends on oxygen pressure during deposition. ${ }^{6)}$ According to their results, $\mathrm{NiO}$ thin films deposited at $1.33 \times$ $10^{-5} \mathrm{~Pa}$ of $\mathrm{O}_{2}$ are amorphous from the point of view of XRD and crystalline from the point of view of RHEED, whereas a $\mathrm{NiO}$ thin film deposited at $1.33 \times 10^{-4} \mathrm{~Pa}$ of $\mathrm{O}_{2}$ is epitaxial by both XRD and RHEED. These results indicate that room temperature epitaxial growth of $\mathrm{NiO}$ thin film is very sensitive to the deposition condition. In our work, the $\mathrm{NiO}$ thin film was deposited at $1.33 \times 10^{-2} \mathrm{~Pa}$ of $\mathrm{O}_{2}$. Therefore, direct comparison of our results with theirs is difficult. It can be inferred that an amorphous thin film examined using XRD can often show in-plane orientation when examined using RHEED.

The NiO thin film resistivity was measured at room temperature for 120-nm-thick thin films. As portrayed in Fig. 4, the deposition rate depends on the magnetic field during deposition, the $\mathrm{NiO}$ thin film deposition was controlled by changing the deposition time. Figure 7 presents the change of resistivity with the magnetic field measured at room temperature. As this figure shows, the $\mathrm{NiO}$ thin film resistivity decreases exponentially with the magnetic field during deposition. For this work, two-point probe method was used to evaluate resistivity. Resistivity $\rho$ of the $\mathrm{NiO}$ thin film was corrected as shown below.

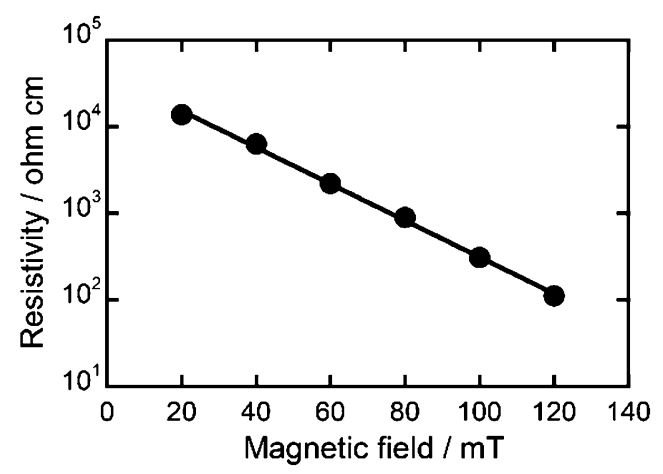

Fig. 7. Change of resistivity of $\mathrm{NiO}$ thin film deposited at room temperature with an applied magnetic field.

$$
\rho=R \frac{w \pi}{\ln 2}
$$

Therein, $R$ and $w$ respectively represent the resistance measured using the two-point probe method and the thin film thickness. For the two-point probe method, the effect of contact resistance must be considered. For this work, the contact resistance was estimated by comparing the calculated resistance with the measured resistance for a 0.25 mm-thick metal Ni sheet. Because the resistivity of metal $\mathrm{Ni}$ at room temperature is $6.84 \times 10^{-6} \Omega \mathrm{cm},{ }^{20)}$ the resistance is calculated as $6.04 \times 10^{-5} \Omega$ based on Eq. (1). The measured resistance obtained using the two-point method was $0.9 \Omega$. The measured resistance is much larger than the calculated value. Therefore, the measured resistance $(0.9 \Omega)$ is believed to correspond to the contact resistance. Figure 7 depicts that the least resistivity is obtained for $\mathrm{NiO}$ thin film deposited under a magnetic field of $120 \mathrm{mT}$. For this film, the resistance value was $2.05 \times 10^{6} \Omega$. Because this resistance value is much larger than the estimated contact resistance, one can neglect the contact resistance effects. The fact that the $\mathrm{NiO}$ thin film resistivity decreases exponentially with the magnetic field suggests that the $\mathrm{Ni}^{3+}$ ion concentration would increase exponentially with the magnetic field during deposition according to the analogy of sputtered $\mathrm{NiO}$ thin films. ${ }^{19)}$ For a sputtered $\mathrm{NiO}$ thin film, the reported resistivity is $0.3-0.8 \Omega \mathrm{cm}$. This magnitude of resistivity is $2-3$ orders lower than that portrayed in Fig. 7. If the $\mathrm{NiO}$ thin film resistivity depends simply on the $\mathrm{Ni}^{3+}$ concentration, then the concentration in a $\mathrm{NiO}$ thin film deposited under the magnetic field is much lower than that in a sputtered $\mathrm{NiO}$ film. Reportedly, Li-doping can control $\mathrm{NiO}$ thin film resistivity. For example, the resistivity of a $0.05 \% \mathrm{Li}$-doped $\mathrm{NiO}$ thin film has been reported as $750 \Omega \mathrm{cm}$ at room temperature. ${ }^{17)}$ This resistivity is almost equal to that of a $\mathrm{NiO}$ thin film deposited under a magnetic field of $80 \mathrm{mT}$. Therefore, results of this study clarified that, without $\mathrm{Li}$-doping, the $\mathrm{NiO}$ thin film resistivity can be controlled by the magnitude of magnetic field application during deposition.

\section{Conclusions}

For this work, PLD along with an electromagnet 
(dynamic aurora PLD) and RHEED was developed by installing an 11-tuned solenoid coil in the vacuum chamber. This method achieved thin film deposition under a magnetic field of up to $120 \mathrm{mT}$ using $1,200 \mathrm{~A}$ of dc current. Because the electromagnet is a solenoid coil, the magnetic field is proportional to the electric field without saturation of the magnetic field. After turning off the electromagnet, but without breaking vacuum, this PLD method can allow in situ observation of the crystal structure of the thin film surface. Many reports of the literature describe studies of PLD in a magnetic field. In most such studies, permanent magnets have been used to apply a magnetic field to the plume. However, in this case, carrying out in-situ observations using RHEED is difficult because the magnetic field deflects the electron beam.

This case study examined the effects of a magnetic field applied to the plume on the in-plane orientation and resistivity of $\mathrm{NiO}$ thin film deposited on $\mathrm{MgO}(001)$ single crystal at room temperature $\left(25^{\circ} \mathrm{C}\right)$. Results demonstrate that the in-plane orientation (epitaxial growth) is unchanged, whereas the thin film resistivity decreases exponentially with the magnetic field during deposition. These findings suggest that the $\mathrm{Ni}^{3+}$ ion concentration can be expected to increase exponentially with the magnetic field during deposition. The room-temperature resistivity of $\mathrm{NiO}$ thin films deposited under a magnetic field of $120 \mathrm{mT}$ was 112 $\Omega \mathrm{cm}$. Results clarified that the $\mathrm{NiO}$ thin film resistivity can be controlled by the magnitude of the magnetic field applied during deposition without Li-doping.

Acknowledgments Part of this work was supported by a Grant-in-Aid for Scientific Research from the Ministry of Education, Culture, Sports, Science and Technology (No. 18H01705). Part of this work was conducted under the Cooperative Research Project Program of the Research Institute of Electronics, Shizuoka University. This research was also supported in part by the Collaborative Research Project of Laboratory for Materials and Structures, Institute of Innovative Research, Tokyo Institute of Technology.

\section{References}

1) L. Dirnberger, P. E. Dyer, S. R. Farrar and P. H. Key, Appl. Phys. A-Mater., 59, 311-316 (1994).
2) M. Takeuchi and T. Kobayashi, Jpn. J. Appl. Phys., 38, 3642-3645 (1999).

3) N. Wakiya, T. Kawaguchi, N. Sakamoto, H. Das, K. Shinozaki and H. Suzuki, J. Ceram. Soc. Jpn., 125, 856-865 (2017).

4) R. Jordan, D. Cole and J. G. Lunney, Appl. Surf. Sci., 109-110, 403-407 (1997).

5) H. Minami, D. Manage, Y. Y. Tsui, R. Fedosejevs, M. Malac and R. Egerton, Appl. Phys. A-Mater., 73, 531534 (2001).

6) M. Tachiki, T. Hosomi and T. Kobayashi, Jpn. J. Appl. Phys., 39, 1817-1820 (2000).

7) T. Kobayashi, H. Akiyoshi and M. Tachiki, Appl. Surf. Sci., 197-198, 284-303 (2002).

8) N. Wakiya, K. Muraoka, T. Kadowaki, T. Kiguchi, N. Mizutani, H. Suzuki and K. Shinozaki, J. Magn. Magn. Mater., 310, 2546-2548 (2007).

9) H. Agura, A. Suzuki, T. Matsushita, T. Aoki and M. Okuda, Thin Solid Films, 445, 263-267 (2003).

10) T. Kanashima, J. M. Park, D. Ricinschi and M. Okuyama, Ferroelectrics, 466, 63-73 (2014).

11) K. Zhang, J. Dai, X. Zhu, X. Zhu, X. Zuo, P. Zhang, L. $\mathrm{Hu}, \mathrm{W}$. Lu, W. Song, Z. Sheng, W. Wu, Y. Sun and Y. Du, Sci. Rep., 6, 19483 (2016).

12) N. Wakiya, N. Sakamoto, S. Koda, W. Kumasaka, N. Debnath, T. Kawaguchi, T. Kiguchi, K. Shinozaki and H. Suzuki, NPG Asia Mater, 8, e279 (2016).

13) N. Debnath, T. Kawaguchi, W. Kumasaka, H. Das, K. Shinozaki, N. Sakamoto, H. Suzuki and N. Wakiya, J. Magn. Magn. Mater., 432, 391-395 (2017).

14) N. Debnath, T. Kawaguchi, H. Das, S. Suzuki, W. Kumasaka, N. Sakamoto, K. Shinozaki, H. Suzuki and N. Wakiya, Sci. Technol. Adv. Mater., 19, 507-516 (2018).

15) Y. Kakehi, S. Nakao, K. Sato and T. Kusaka, J. Cryst. Growth, 237-239, 591-595 (2002).

16) A. Yang, O. Sakata, R. Yamauchi, Y. Katsuya, L. S. R. Kumara, Y. Shimada, A. Matsuda and M. Yoshimoto, Appl. Surf. Sci., 320, 787-790 (2014).

17) D. Adler and J. Feinleib, Phys. Rev. B, 2, 3112-3134 (1970).

18) W. L. Jang, Y. M. Lu, W. S. Hwang, T. L. Hsiung and H. P. Wang, Appl. Phys. Lett., 94, 062103 (2009).

19) Y. M. Lu, W. S. Hwang and J. S. Yang, Surf. Coat. Tech., 155, 231-235 (2002).

20) P. S. Neelakanta, "Handbook of Electronic Materials", CRC Press, Boca Raton Florida (1995) pp. 215-216. 